\title{
Four Station Knee Simulator Wear Testing Comparing Titanium Niobium Nitride with Cobalt Chrome
}

\section{Malikian R, Maruthainar $\mathrm{K}^{*}$, Stammers J, Wilding CP and Blunn GW}

John Scales Centre for Biomedical Engineering, The Institute of Orthopaedics and Musculoskeletal Science, Royal National Orthopaedic Hospital Trust, Brockley Hill, Stanmore, Middlesex, UK

\begin{abstract}
A new non-destructive method was applied in order to assess bone integrity. The method is based on measurement of bHypersensitivity to an orthopaedic implant was first published in 1966 and since then, in sensitive patients, is known to cause serious problems in joint replacement surgery. Titanium niobium nitride (TiNbN) can act as a surface coat for knee arthroplasty to "hide" the cobalt chrome (CoCr) femoral component beneath, therefore affording an immunoprivileged state. The aim of this study is to determine the wear properties of titanium niobium nitride against Ultra High Molecular Weight Polyethylene (UHMWPE) compared to cobalt chrome and to examine the metallic alloy surface of knee prostheses after loading cycles using a knee simulator. Three TiNbN coated and one CoCr Vanguard total knee femoral components were articulated against standard UHMWPE grade tibial inserts in the Stanmore-Instron knee simulator. Surface roughness, UHMWPE mass, lowest point, surface profiles and volumetric change were measured every one million cycles up to five million cycles. After five million cycles the average roughness of the cobalt chrome medial and lateral femoral condyles was over three times that of the TiNbN coated femoral condyles. There was no obvious difference in weight loss, volume loss or progression of lowest points of the tibial inserts articulating with the $\mathrm{TiNbN}$ coated and the cobalt chrome femoral component. Despite a clear reduction in roughness progression over the course of this in vitro test, there was no demonstrable improvement in UHMWPE wear measured gravimetrically or by surface profiling. The TiNbN implant tested may still be of great benefit to patients who are metal sensitive, but the coat offers no benefit in UHMWPE wear.
\end{abstract}

Keywords: Knee arthroplasty; Titanium niobium nitride; Knee simulator; Wear

\section{Introduction}

Hypersensitivity to an orthopaedic implant was first published in 1966 [1] and since then it was suggested it can pose serious problems in joint replacement surgery. $10-20 \%$ of the general population is reported to have metal sensitivity [2-4]. These patients are susceptible to type IV hypersensitivity whereby the release of nickel, cobalt and chromium ions in their cobalt chrome $(\mathrm{CoCr})$ implantcan cause a cell-mediated immune reaction [5]. There is still debate within the orthopaedic community about the presence and significance of deep metal sensitivity associated with orthopaedic implants. However, many patients complain about pain with no radiographic evidence of implant malpositioning and the diagnosis of metal sensitivity is often based just on positive skin patch tests. This involves application of the potential allergen to bare skin and the positive identification of a contact dermatitis reaction within 48 hours, effectively inducing a type IV hypersensitivity reaction on the skin. Additionally patients requiring knee joint replacements often indicate that they are metal sensitive and this is associated with a positive skin patch test.

To address this issue, various strategies possible solutions have been investigated: the CoCr femoral components can be coated or alternative femoral component materials such as ceramics can be used. Titanium niobium nitride (TiNbN) can act as a surface coat to "hide" the cobalt chrome femoral component beneath, therefore affording an immunoprivileged state [6]. Titanium niobium nitride is a coating applied by physical vapour deposition (PVD). PVD is a process by which positively charged metal ions are evaporated in a vacuum chamber and react with inert gases introduced to the chamber. The surfaces that are to be coated are negatively charged to allow a strong bond to form between the substrate and the coating $[7,8]$.

The aim of this study is to determine whether there is a difference in Ultra High Weight Polyethylene (UHMWPE) wear articulating against cobalt chrome and titanium niobium nitride and to examine the metallic alloy surface after 5 million loading cycles in a knee simulator.

\section{Materials and Methods}

Four CoCr Vanguard (Biomet, Bridgend, UK) total knee replacements were used. Three femoral components were coated with TiNbN via physical vapour deposition, the other made from CoCr. Four corresponding tibial base-plates were all made of $\mathrm{CoCr}$. Six implant grade UHMWPE tibial inserts of $10 \mathrm{~mm}$ were used; four in the simulator wear testing, and two as soak controls.

The Stanmore-Instron four station, force driven knee simulator was used, providing six degrees of freedom of motion (Anterior/Posterior, Lateral/Medial, Superior/Inferior) in accordance with ISO standard 14243 providing proven clinical wear performance. The components were fixed using Simplex P, acrylic bone cement (Stryker Orthopaedics, Mahwah, NJ, USA) to the simulator. Before commencing the test all the components were aligned. The femoral and tibial components were aligned to $0^{\circ}$ of flexion and $0^{\circ}$ antero-posterior tilt, varus/valgus and axial rotation respectively. The knee simulator was run at $1 \mathrm{~Hz}$ using

*Corresponding author: Kunalan Maruthainar, John Scales Centre for Biomedical Engineering, The Institute of Orthopaedics and Musculoskeletal Science, Royal National Orthopaedic Hospital Trust, Brockley Hill, Stanmore, Middlesex, HA7 4LP, UK, Tel: 02088954 2300-5427/5378; E-mail: kunalan@gmail.com

Received July 11, 2013; Accepted August 24, 2013; Published August 30, 2013

Citation: Malikian R, Maruthainar K, Stammers J, Wilding CP, Blunn GW (2013) Four Station Knee Simulator Wear Testing Comparing Titanium Niobium Nitride with Cobalt Chrome. J Bioengineer \& Biomedical Sci 3: 125. doi: 10.4172/21559538.1000125

Copyright: @ 2013 Malikian R, et al. This is an open-access article distributed under the terms of the Creative Commons Attribution License, which permits unrestricted use, distribution, and reproduction in any medium, provided the original author and source are credited. 
a standard walking cycle at a temperature of $37^{\circ} \mathrm{C}$. Figure 1 illustrates the load, torque, displacement, rotation, soft tissue load and anteriorposterior shear load throughout the simulated gait cycle.

Prior to commencing testing and after completion of each million cycles all components were cleaned under aseptic conditions in Decon (Decon Laboratories Limited East Sussex, UK) and washed with distilled water to remove lubricant and proteins. They were placed in an incubator at $37^{\circ} \mathrm{C}$ to dry for 24 hours.

The weight of UHMWPE inserts was measured using a Precisa 92SM-202A scale (Precisa Instruments AG, Dietikon, Switzerland) accurate to 5 decimal points. In order to keep fluid absorption into consideration the two soak controls were weighed and the net difference was calculated at each cycle interval. If a mass difference in the controls was measured it would be used to correct the mass of the test components to ensure any changes were only due to wear. The surface profile of the tibial inserts were measured using an NPL soft probe profiler (3D Digital Design, Southgate, England) controlled by a computer programme driven machine (Heidenhain, West Sussex, England). Prior to testing, three indentations were milled around the edges of the polyethylene inserts in areas away from the wear tracks. This act as calibration start points for the surface profiler to ensure the same area is identified over repeated tests for consistent analysis without affecting the wear profile itself. This procedure was performed to allow the profiler to accurately measure changes in the surface of the insert over the course of simulator wear testing. By subtracting the interval profiles from the pre-test map, the volumetric change during the test could be derived. The lowest points on the medial and lateral sides of inserts were measured using a hand operated measuring gauge and validated against ISO 14243 standards.

As suggested in the ISO 14243 standards, a lubricant of 30\% fetal
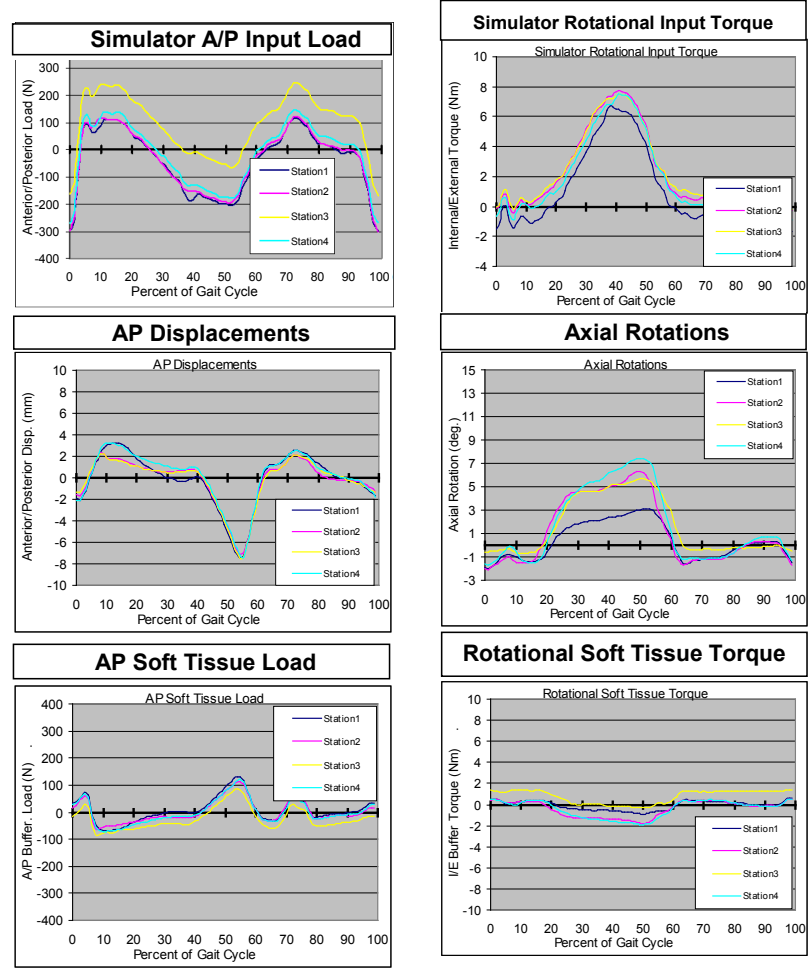

Figure 1: Graphs demonstrating the forces and movements in the simulator at 5 million cycles. calf serum and $0.8 \%$ sodium azide was used. The soak controls were held at $37^{\circ} \mathrm{C}$. Apart from the motion and loading, they were subjected to the same treatment as the test samples. The serum was changed every five days and disposed of. The test was run for five million cycles, which is similar to five years total knee replacement use in vivo [9].

Average surface roughness ( $\mathrm{Ra}$ ) was measured using a Mitutoyo Surftest (Mitutoyo, Kawasaki, Japan) with a $0.08 \mathrm{~mm}$ cut-off. Six measurements were taken at four points perpendicular to the anteriorposterior plane on the medial and the lateral condyle. These were at $10^{\circ}$ intervals from $0^{\circ}$ to $30^{\circ}$ flexion. This resulted in 24 measurements per femoral component, from which an average roughness was calculated.

\section{Results}

\section{Surface roughness}

Prior to the start of the test, the average roughness of the titanium niobium nitride implants ( 0.035 micrometers) was greater than that of the cobalt chrome implants (0.018 micrometers), although there were no visible scratches. At the end of the test the average roughness of the cobalt chrome medial and lateral femoral condyles was a factor of 2.23 and 4 greater than that of the average roughness of the titanium niobium nitride coated femoral condyles respectively. $\mathrm{CoCr}$ lateral condyle roughness increased over the five million cycles by a factor of 17.41 from 0.017 to 0.296 . The corresponding change for the titanium niobium nitride coated lateral condyles was from 0.036 to 0.074 , equivalent to a rise of a factor of 2.04 . The cobalt chrome medial condyle roughness rose from 0.019 to 0.256 resulting in an increase by a factor of 13.69. The titanium niobium nitride coated medial femoral condyle average roughness rose over the course of the test from a start figure of 0.034 to 0.115 . This equated to a rise of a factor of 3.37 . The difference in average roughness was greater for the lateral condyle than the medial condyle. The graphical representations of the roughness are presented in Figure 2.

$\mathrm{CoCr}$ femoral condylar surface roughness had a relatively symmetrical distribution, with bimodal peaks of greatest surface roughness in the middle region of both medial and lateral condyles. All Titanium niobium nitride condyles had an asymmetrical distribution of surface roughness, with greatest surface roughness being in the medial condyles, seen as an obvious spike on the graph in Figure 3.

\section{Gravimetric wear}

There was no obvious difference in the weight loss of the tibial inserts articulating with the titanium niobium nitride coated and the cobalt chrome femoral components. The mean weight loss of the three titanium articulating inserts was $0.04222 \mathrm{~g}$ over five million cycles,

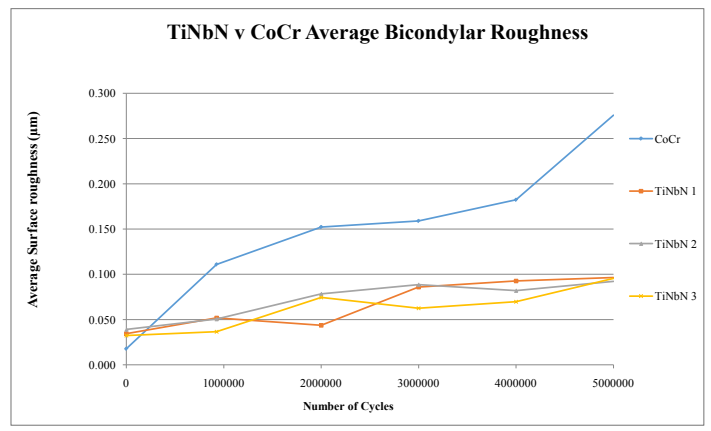

Figure 2: Average femoral roughness. 


\section{CoCr surface roughness with position} along condyle

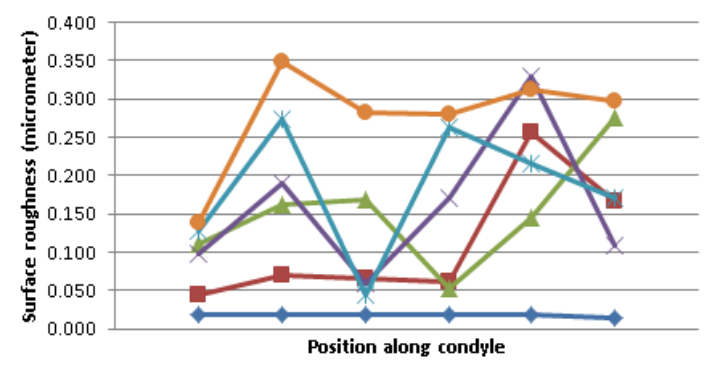

TiN (2) surface roughness with position along condyle

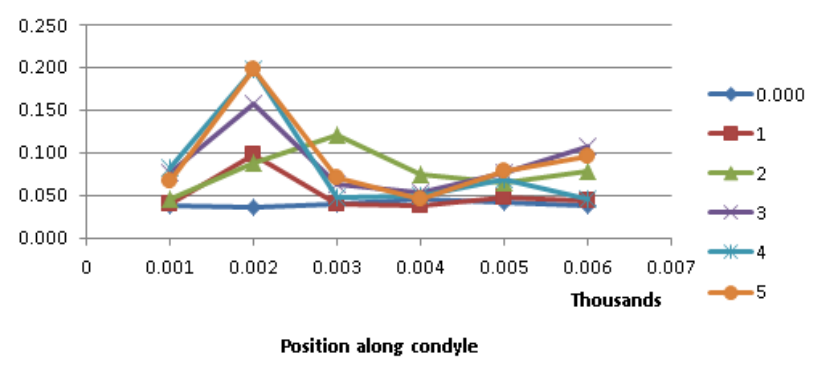

\section{TiN (1) surface roughness with position} along condyle

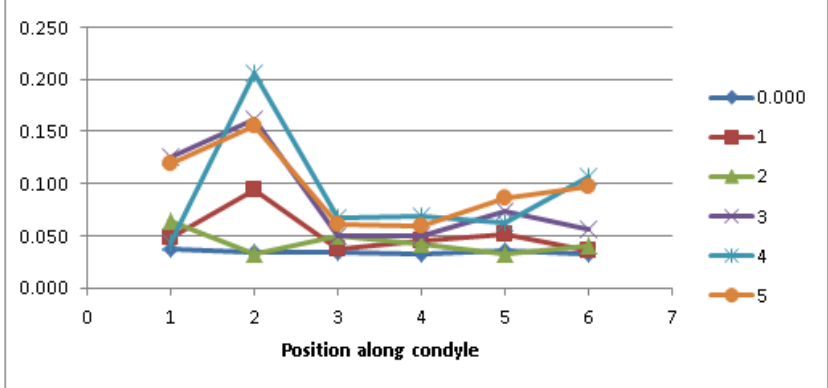

\section{TiN (3) surface roughness with position} along condyle

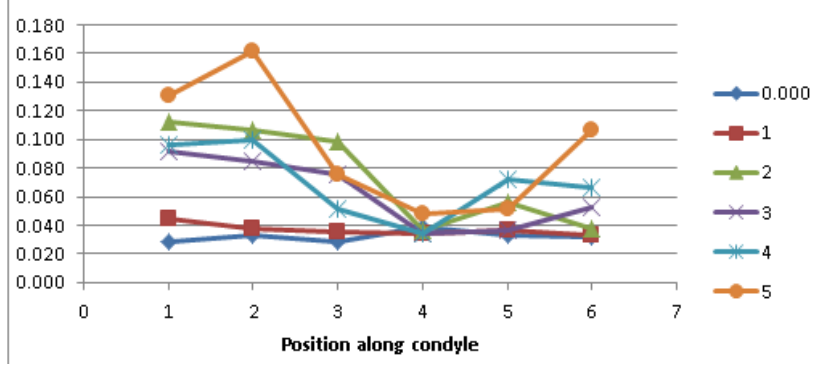

Figure 3: Graphs demonstrating relationship between surface roughness and position along knee replacement component.

whereas, for the one insert articulating against cobalt chrome was $0.02611 \mathrm{~g}$ over the test period. At 2,000,000 cycles there was a negative mass change with the TiNbN 3 component, indicating mass. The gravimetric wear of the individual inserts is presented in Figure 4.

\section{Surface profiling}

The results of the surface profiling which were used to calculate volume loss of UHMWPE showed no clear difference between the two bearing surfaces (Figure 5). There was no evidence of a reduction in volume loss with a titanium niobium nitride surface.

\section{Lowest points}

The lowest points show a downward progression over the time of the simulation. There was no significant difference between the progression of the lowest points of the titanium niobium nitride coated and the cobalt chrome over the course of the test (Figure 6 medial, Figure 7 lateral).

\section{Discussion}

\section{Surface roughness of femoral components}

After five million cycles in the knee simulator the surface roughness of the three TiNbN coated components was lower than the post-test roughness of the cobalt chrome coated component. This is despite the CoCr component startingwith a pre-test roughness less than the TiNbN components.

From the measurements, the TiNbN appears to exhibit a scratch resistance greater than that of cobalt chrome. This finding is in accordance with work in pin on plate, and knee simulators [1012]. The medial roughness rose from 0.034 to 0.115 for the titanium components, compared to a rise from 0.019 to 0.256 for the cobalt chrome. The scratch resistance of titanium niobium nitride has been demonstrated before in a hip simulator [7]. It must be noted that there was a greater difference in average roughness progression for the lateral condyles when compared to the medial. The average roughness for the CoCr lateral condyle increased over the five million cycles from 0.017 to 0.296, whilst the titanium lateral condyles changed from 0.036 to 0.074 . The average roughness assessed across both condyles rose from 0.018 to 0.276 for the CoCr by the end of the test. Such a rise was far higher than that of the TiNbN rising from 0.0353 to 0.095 . The difference between the medial and lateral components may be due to the direction of measurement relative to the direction of sliding during testing. Measurements were taken perpendicular to the anterior-posterior sliding and may over-estimate the roughness. Taking an average of 24 recordings for each condyle aimed to reduce the significance of error.

It is important to note that the influence of surface roughness on wear is minimal between 0.15 and 0.28 micrometers. After 5 million cycles only the CoCr surface roughness was greater than this at 0.296 on the medial condyle.

The use of a single cobalt chrome component was not optimum, however the decision to use three TiNbN and only one CoCr control in a four station knee simulator was based on the ISO14243 standards requiring analysis of three samples of the test material. At the end of five million cycles the titanium niobium nitride coat was intact to the naked eye conferring that it was retained [13-16]. Further experiments would have extra cobalt chrome controls to increase the power and enable 


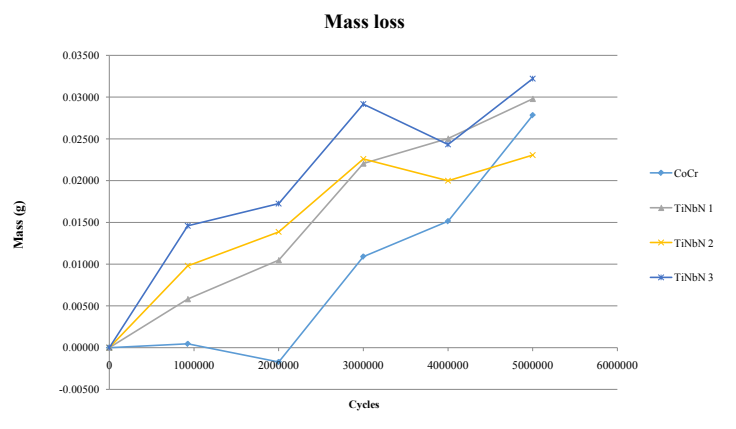

Figure 4: Graph of mass loss of tibial inserts involved in simulator wear testing

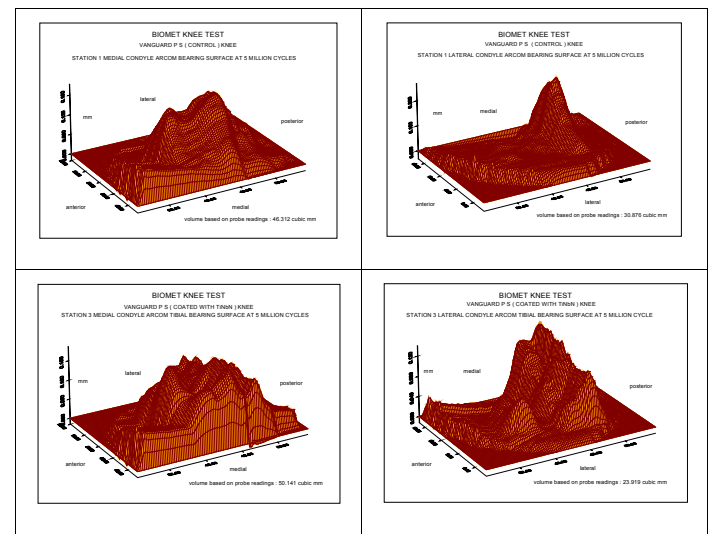

Figure 5: NPL 3D wear plots showing area of wear above the plane.

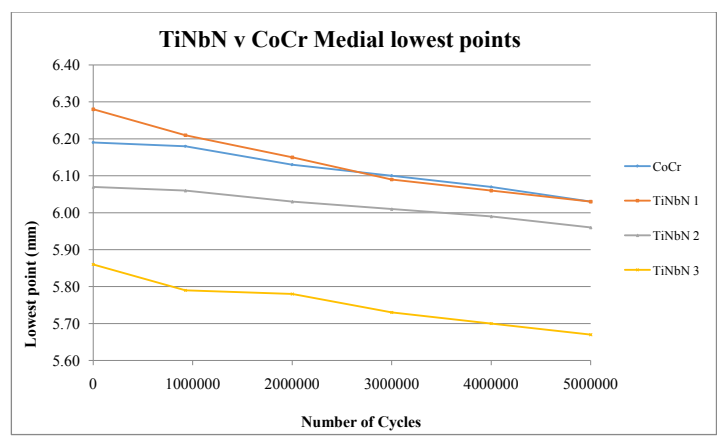

Figure 6: Graph of the lowest points of medial condyles.

further statistical analysis to determine the significance of difference found.

\section{Wear of UHMWPE inserts}

Despite the reduction in roughness progression noted for the titanium components, the gravimetric wear was similar to that of the cobalt chrome component tested. In similar knee simulator studies using zirconium oxide bearing surfaces, the scratch resistance of the surface was matched by an improvement in weight loss [17]. The higher friction of the surface of titanium niobium nitride may increase adhesive wear. The UHMWPE showed no signs of delamination and therefore the reason for the wear was probably adhesive. Scratches due to sliding may have initiated rapid UHMWPE wear. It could be assumed that implants with lower scratch resistance would be more susceptible to abrasive than adhesive wear. Analysis of the wear particles within

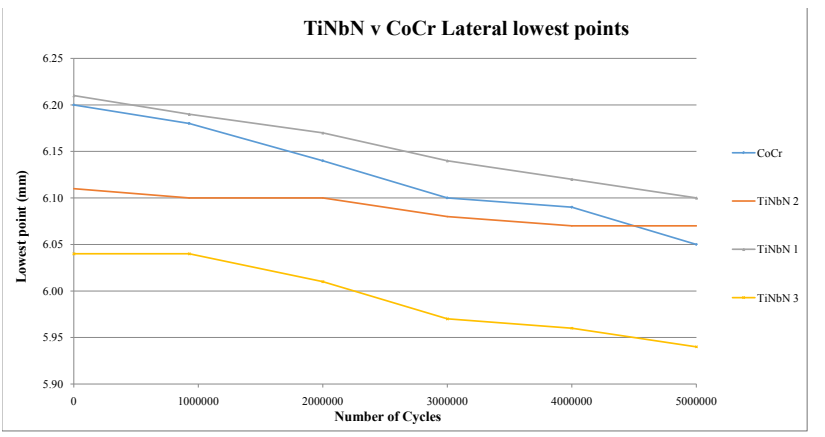

Figure 7: Graph of the lowest points of lateral condyles.

the serum or scanning electron microscopy of UHMWPE may have provided evidence for the specific wear mechanism.

\section{Surface of the UHMWPE tibial inserts}

Analysis of the surface of the UHMWPE did not reveal any differences between the volumetric wear of UHMWPE of the titanium and cobalt chrome. When interpreting surface profiling, creep must always be considered to be a cause of possible bias.

\section{Conclusion}

Despite a clear reduction in roughness progression over the course of this in vitro test, there was no demonstrable improvement in UHMWPE wear measured gravimetrically and by surface profiling. The implant tested may still be of great benefit to patients who are metal sensitive, but the coat offers no benefit in UHMWPE wear. The implant has not been marketed as an alternative to cobalt chrome in those that suffer severe knee osteoarthritis but as an option to patients with metal ion allergies.

\section{Acknowledgement}

We are indebted to Mr Bob Skinner, Mark Harrison and Keith Rayner for their hard work for this study.

\section{Conflict of interest}

Supported in part by a research grant funded by Biomet. Biomet had no role in the study design, analysis or manuscript content. The authors have no affiliation with the funding company.

\section{References}

1. Foussereau J, Laugier P (1966) Allergic eczemas from metallic foreign bodies Trans St Johns Hosp Dermatol Soc 52: 220-225.

2. Elves MW, Wilson JN, Scales JT, Kemp HB (1975) Incidence of metal sensitivity in patients with total joint replacements. Br Med J 4: 376-378.

3. Nasser S (2007) Orthopedic metal immune hypersensitivity. Orthopedics 30 89-91.

4. Niki Y, Matsumoto $H$, Otani T, Yatabe T, Kondo M, et al. (2005) Screening for symptomatic metal sensitivity: a prospective study of 92 patients undergoing total knee arthroplasty. Biomaterials 26: 1019-1026.

5. Ingham E, Fisher J (2000) Biological reactions to wear debris in total joint replacement. Proc Inst Mech Eng H 214: 21-37.

6. Munro-Ashman D, Miller AJ (1976) Rejection of metal to metal prosthesis, skin sensitivity to cobalt. Contact Dermatitis 2: 65-67.

7. Pappas MJ, Makris G, Buechel FF (1995) Buechel. Titanium nitride ceramic film against polyethylene. A 48 million cycle wear test. Clin Orthop Relat Res 64-70.

8. Nevelos J (2004) Surface engineering of prosthetic knee components. Knee 11: $163-167$. 
Citation: Malikian R, Maruthainar K, Stammers J, Wilding CP, Blunn GW (2013) Four Station Knee Simulator Wear Testing Comparing Titanium Niobium Nitride with Cobalt Chrome. J Bioengineer \& Biomedical Sci 3: 125. doi: 10.4172/2155-9538.1000125

Page 5 of 5

9. Schmalzried TP, Szuszczewicz ES, Northfield MR, Akizuki KH, Frankel RE, et al. (1998) Quantitative assessment of walking activity after total hip or knee replacement. J Bone Joint Surg Am 80: 54-59.

10. Sawase T, Yoshida K, Taira Y, Kamada K, Atsuta M, et al. (2005) Abrasion resistance of titanium nitride coatings formed on titanium by ion-beam-assisted deposition. J Oral Rehabil 32: 151-157.

11. Kamali A, Farrar R, Hatto P, Stone MH, Fisher J (2005) Wear of ultrahighmolecular weight polyethylene against titanium-nitride-coated-countersurfaces. Proc Inst Mech Eng Part J Journal of Engineering Tribiology 219: 41-47.

12. Peterson CD, Hillberry BM, Heck DA (1988) Component wear of total knee prostheses using Ti-6A1-4V, titanium nitride coated Ti-6A1-4V, cobaltchromium-molybdenum femoral components. J Biomed Mater Res 22: 887-903.

13. Rodriguez D, Manero JM, Gil FJ, Planell JA (2001) Low cycle fatigue behavior of Ti6Al4V thermochemically nitrided for its use in hip prostheses. J Mater Sci Mater Med 12: 935-937.

14. Satomi K, Akagawa $Y$, Nikai $H$, Tsuru $H$ (1988) Tissue response to implanted ceramic-coated titanium alloys in rats. J Oral Rehabil 15: 339-345.

15. Scarano A, Piattelli M, Vrespa G, Caputi S, Piattelli A (2003) Bacterial adhesion on titanium nitride-coated and uncoated implants: an in vivo human study. J Oral Implantol 29: 80-85.

16. Khan MA, Williams RL, Williams DF (1999) Conjoint corrosion and wear in titanium alloys. Biomaterials 20: 765-772.

17. Lee JK, Maruthainar K, Wardle N, Haddad F, Blunn GW (2009) Increased force simulator wear testing of a zirconium oxide total knee arthroplasty. The Knee 16: $269-274$. 